\title{
3D Finite Element Modeling of Free-Surface Flows with Efficient $k-\epsilon$ Turbulence Model and Non-hydrostatic Pressure
}

\author{
Célestin Leupi ${ }^{1}$ and Mustafa Siddik Altinakar ${ }^{2}$ \\ 1 ISE-STI-LIN, Ecole Polytechnique Fédérale, \\ Lausanne 1015, Switzerland \\ Phone: +41.21.693.25.07, Fax + 41.21.693.36.46 \\ celestin.leupi@epfl.ch \\ 2 NCCHE, The University of Mississipi, \\ Carrier Hall Room 102 University, MS 38677 USA
}

\begin{abstract}
Validation of 3D finite element model for free-surface flow is conducted using a high quality and high spatial resolution data set. The present research finds its motivation in the increasing need for efficient management of geophysical flows such as estuaries (multiphase fluid flow) or natural rivers with the complicated channel geometry (e.g. strong channel curvature). A numerical solution is based on the unsteady Reynolds-averaged Navier-Stokes equations without conventional assumption of hydrostatic pressure. The model uses implicit fractional step time stepping, with the characteristic method for convections terms . The eddy viscosity is calculated from the efficient $k-\epsilon$ turbulence model. The RANS are solved in the multi-layers system (suitable for the vertical stratified fluid flow ) to provide the accurate resolution at the bed and free-surface. The model is applied to the 3D curved open channels flows for which experimental data are available for comparison. Good agreement is found between numerical computations and experiments.
\end{abstract}

Keywords: Validation; Characteristic method; 3D Curved open channel; secondary currents; Non-hydrostatic pressure.

\section{Introduction}

Nowadays with the increasing computer power, several 3D computations have been successfully conducted for geophysical flows modeling. Most of these models have used the conventional hydrostatic pressure assumption. However, the natural rivers mostly have complicated geometry with the strong channel curvature. Such flows are of more importance for environmental hydraulic engineering and some related important features such as the secondary flows generated by the channel curvature and the related background turbulence effects, need to be well understood while a 3D description of the velocity field is required. Thus it is useful to resort to a more accurate model in which the hydrostatic assumption 
is removed. Nevertheless, the importance of non-hydrostatic pressure in computational fluid problems was demonstrated and many researchers have applied 3D non-hydrostatic models to simulate the curved open channel flows. Wu et al. [13 and Olsen [10] used 3D numerical models to study the flow structure and mass transport in curved open channel. Xiabo et al. 2 have simulated the $3 \mathrm{D}$ unsteady curved open channel with standard $k-\epsilon$ turbulence model and the non-hydrostatic pressure on the conformal mesh, but conformal mesh could poorly performed for some complicated bathymetry. Lai et al. [4 have used finite volume method on the unstructured grid to simulate $3 \mathrm{D}$ flow in meandering channel. For the free surface treatment, most of these 3D models employed the rigid-lid approximation, which have some weaknesses especially in strongly curved open channel flows (see [5]).

Based on the novel approach developed by Leupi et al. [6], the present model adopts the finite element conservative formulation in the multi-layers system for providing an accurate resolution at the bed and the free-surface.

The present work aims at validating the 3D finite element model against well-known non-uniform and unsteady flows in curved open channel flows using a high quality and high spatial resolution data set. The model uses the nonhydrostatic pressure and the state-of-art $k-\epsilon$ turbulence model closure to solve the Reynolds-averaged Navier-Stokes equations (RANS).

In this study, the free-surface movement is controlled through the so-called integrated continuity equation. The full 3D governing equations are solved using implicit fractional time marching stepping where final velocity field and pressure term are computed from the hydrodynamic correction. Euler or Runge-Kutta scheme is used to obtain a set of algebraic equations from discretization. An efficient fractional step algorithm from Mohammadi and Pironneau [9] is introduced for the $k-\epsilon$ model. This paper deals with the simulation of the 3D turbulent flow in the open curved channel for which experimental data are available.

\section{Governing Equations}

Let us consider an incompressible fluid body in a three-dimensional time varying domain $\widehat{\Omega}$ (see also [6]) with $\Omega$ the projection of $\widehat{\Omega}$ on the $x y$ horizontal plane. $\widehat{\Omega}$ is bounded by the free-surface $\Gamma_{s}$ given by $z=\eta(x, y, t)$, the bottom topography $\Gamma_{b}$ given by $z=-\mathfrak{h}(x, y)$, the open boundary denoted by $\Gamma_{o}$. Where $\mathfrak{h}(x, y)$ is the distance between the bottom and the reference plane $x y$ and $\eta(x, y, t)$ the elevation of the free-surface with the respect to the horizontal plane $x y$. For description of the turbulent motion, the pressure $p$ can be written as the sum of an hydrostatic term $p_{h}$ and an hydrodynamic correction $p_{n h}=\rho \tilde{p}$,

$$
p(\mathbf{x}, t)=p_{h}+p_{n h}=p_{a}+g \rho_{o}(\eta-z)+g \int_{z}^{\eta} \Delta \rho \mathrm{d} z+\rho \tilde{p}(\mathbf{x}, t)
$$

The 3D non hydrostatic Reynolds Averaged Navier-Stokes (RANS) equations reads 


$$
\begin{gathered}
\nabla_{H} \cdot \mathbf{U}+\frac{\partial w}{\partial z}=0 \\
\frac{D \mathbf{U}}{D t}+g \nabla_{H} \eta-\nabla_{H}\left(\nu_{T} \nabla_{H} \mathbf{U}\right)-\frac{\partial}{\partial z}\left(\nu_{T} \frac{\partial \mathbf{U}}{\partial z}\right)+g \nabla\left(\int_{\mathfrak{h}}^{\eta} \frac{\Delta \rho}{\rho_{o}} \mathrm{~d} z\right)+\frac{1}{\rho} \nabla \tilde{p}=\mathbf{F}_{\mathbf{x y}} \\
\frac{D w}{D t}-\nabla_{H}\left(\nu_{T} \nabla_{H} w\right)-\frac{\partial}{\partial z}\left(\nu_{T} \frac{\partial w}{\partial z}\right)+\frac{1}{\rho} \frac{\partial \tilde{p}}{\partial z}=0 \\
\frac{\partial \eta}{\partial t}+\nabla_{H} \cdot \int_{-\mathfrak{h}}^{\eta} \mathbf{U d} z=0
\end{gathered}
$$

where $\mathbf{U}=(u, v)^{T}$ is the horizontal velocity vector, $\mathbf{F}_{x y}=(\mathrm{f} v,-\mathrm{f} u)^{T}$ is vector of body forces with $f$ the Coriolis parameter, $g$ is the gravitational acceleration, $\nu_{T}$ is the eddy viscosity, (see Rodi [12]). $(\nabla \cdot)$ is the $3 D$ divergence operator, $\frac{D}{D t}$ represents the material derivative, and $\left(\nabla_{H^{*}}\right)$ stands as the $2 \mathrm{D}$ horizontal divergence operator. $\Delta \rho=\rho-\rho_{0} ; \rho, \rho_{0}$ are respectively the fluid density and the basic water density.

In eq.(3), the vertical eddy viscosity is defined as

$$
\nu_{T}=\nu+c_{\mu} \frac{k^{2}}{\varepsilon}
$$

in which $\nu$ is the kinematic viscosity.

The $k-\varepsilon$ trubulence equations read $(9])$,

$$
\begin{aligned}
& \frac{D k}{D t}-\nabla \cdot\left[c_{\mu} \frac{k^{2}}{\varepsilon} \nabla k\right]=c_{\mu} \frac{k^{2}}{\varepsilon} G-\varepsilon \\
& \frac{D \varepsilon}{D t}-\nabla \cdot\left[c_{\varepsilon} \frac{k^{2}}{\varepsilon} \nabla \varepsilon\right]=\frac{c_{1}}{2} k G-c_{2} \frac{\varepsilon^{2}}{k}
\end{aligned}
$$

The turbulent constants are given: $c_{1}=0.126, c_{2}=0.07, c_{\mu}=0.09, c_{\varepsilon}=1.92$.

The production is represented by the squared shear frequency, $G$, (see [7]), such as :

$$
G=\frac{1}{2}\left(\|\nabla \mathbf{V}\|+\|\nabla \mathbf{V}\|^{T}\right)^{2}
$$

where $\|$.$\| stands as the Euclidian norm, \mathbf{V}=\mathbf{V}(u, v, w)$ is the $3 \mathrm{D}$ velocity vector.

The depth-integrated continuity equation eq. (5) allow the model to follow the free-surface position. This equation is obtained by integrating the (local) continuity equation (2) in the $\mathrm{z}$ direction using the suitable kinematic free-surface and bottom boundary conditions. 
The horizontal velocity is approximated combining the lowest order RaviartThomas element $\left(\mathbb{R T}_{0}\right)$ in $x y$ plane with the $\mathbb{P}_{1}$ elements along the vertical direction (see [6], 8], 11]). To discretize the convective term a method based on a Lagrange-Galerkin (or characteristics Galerkin) approach is considered (see [8, 9] ) using either Euler scheme or more accurate Runge-Kutta. At each time step it is only required to solve a set of the positive definite symmetric and tridiagonal matrices for the fluxes using the conjugate gradient solver. For the turbulence modeling, the combinaison of the characteristics method with the fractional time stepping algorithm from Mohammadi and Pironneau (9], [] $)$ can allow to preserve the positivity of $k, \varepsilon$ as well as the stability of the scheme (see [9, 12]). To avoid spurious numerical oscillations, the source term, G, has been discretised explicitly while the sink term has been discretised using the quasiimplicit forms with the consequence that linear terms are linearized (see [9]).

\section{$3 \quad$ Numerical Results}

The present model has been applied for simulating a 3D curved open channel flow in Figure 1, for which experimental data are available (see Blanckaert 1]). The

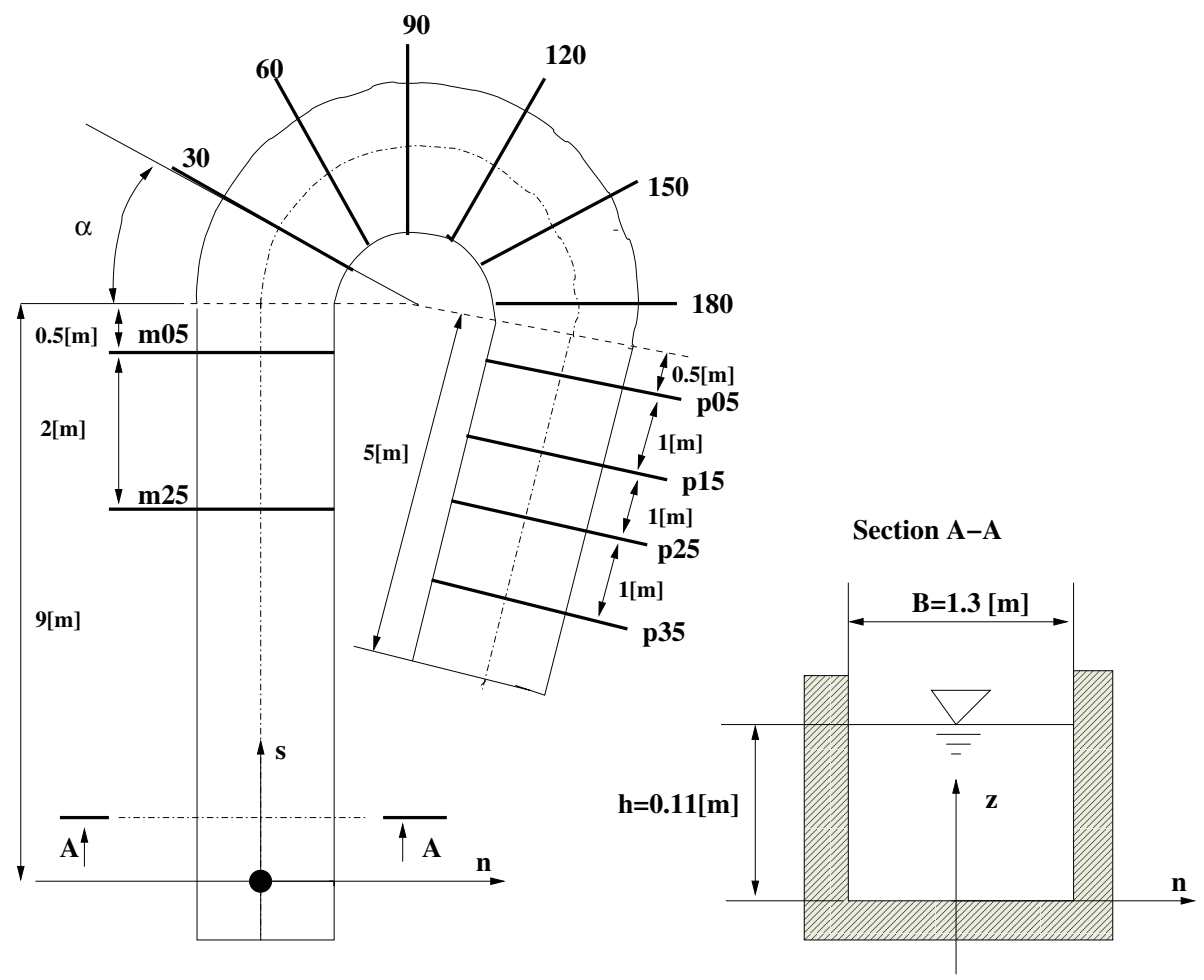

Fig. 1. A $193^{\circ}$ Curved open-channel 


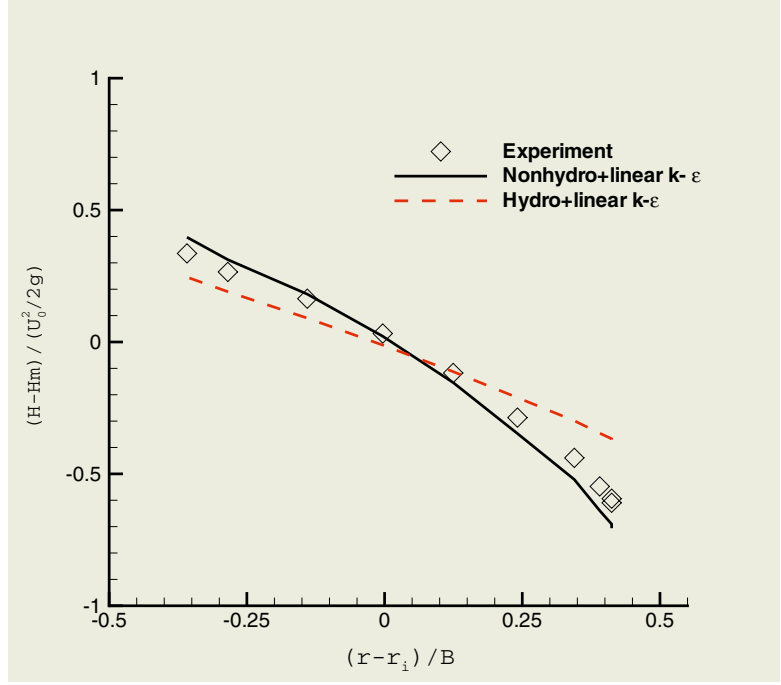

Fig. 2. Experiments versus computed free-surface solutions at section $\alpha=120^{\circ}$ using linear $k-\epsilon$ Turbulence model : Experiment $(\diamond)$; Hydrostatic (dashed line) ; Non hydrostatic (solid line)

discharge is set to $Q=0.089\left[\mathrm{~m}^{3} / \mathrm{s}\right]$, the bed slope (i.e. channel bottom slope) is $S_{0}=0.000624$. The rough bed is characterized by an equivalent roughness height, $k_{s}=0.0022[\mathrm{~m}]$. The flow depth at the outflow (downstream end of the flume) is $0.159[\mathrm{~m}]$. The grid is composed of 50.000 elements and 30.000 nodes. The time step is set to $0.1[\mathrm{~s}]$, and the computation is performed till the flow is well developed at $\mathrm{T}=1300[\mathrm{~s}]$. Computations were conducted with the hydrostatic pressure assumption for different cross-sections with the curvature increasing, to determine the conditions where the non-hydrostatic pressure component become significant. Computed solutions predict the gross flow features, whereas the water surface profile is under-estimated at the out bank, and over-estimated at the inner bank.

Figure2 shows the cross-section at $\alpha=120^{\circ}$, where are found the weaknesses of the hydrostatic pressure solution. Hence agreement with experiments is rather better for non-hydrostatic solutions particularly with the increasing curvature. This suggest that the separation may occur in the vertical flow and the the pressuredriven secondary effects are important. Thus the free surface must be more accurately computed to accounts for its damping effects on the turbulent flow. As observed in figure 3. both hydrostatic and non-hydrostatic pressure solutions show only one secondary flow circulation rotating clockwise from inner bank to outer bank. These predictions do not capture sufficiently the magnitude of the secondary motion. The maximum under prediction in the secondary currents for each vertical examined in this cross-section is ranged between 25 and $95 \%$ for the nonhydrostatic solutions and between 30 and $105 \%$ for the hydrostatic pressure. In Figure 3 the center of vortex is located at about $z=0.25[\mathrm{~m}]$ for the hydrostatic, about $z=0.35[\mathrm{~m}]$ for the non hydrostatic solutions which is more closed to experiments located at $z=0.4[\mathrm{~m}]$. The predicted secondary currents intensities are weaker than 

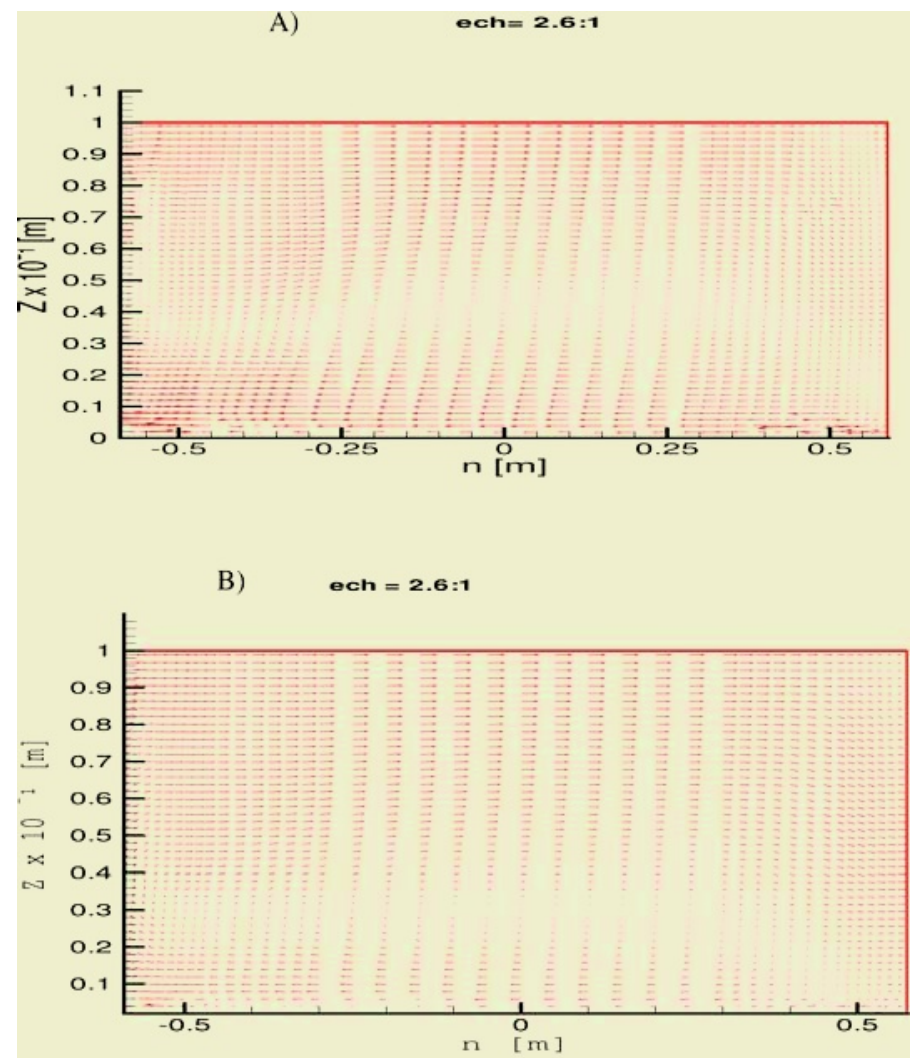

C)

ech $=2.6: 1$

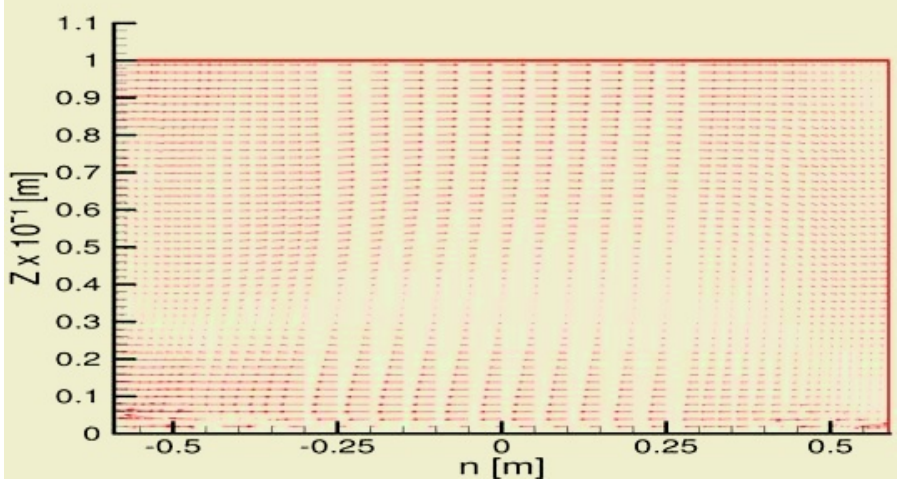

Fig. 3. Experiments versus computed solutions of the of cross-stream velocity vector at section $\alpha=120^{\circ}$ : A) Experiment ; B) Hydrostatic ; C) Non hydrostatic 
measured, and the transverse velocities are under predicted. The anisotropic stress caused by walls and the junction region is not captured by the model, and the reduced momentum is transfered towards the outer region of the bend as well as the position of the longitudinal velocity maximum. This suggest that the turbulencedriven secondary effects are non-linear. Consequently, as shown by Gatsky et al. [3], the related weaker turbulence-driven secondary motion cannot be reproduced by linear and isotropic eddy-viscosity turbulence models. It should be pointed out that the major flow features such as the presence and rotational sense of the major secondary currents are reproduced and agrees well with experiments. The nonhydrostatic pressure influence is found to be more significant with the increasing curvature region, and although being more expensive, it become useful. This suggest that the pressure-driven secondary currents is relatively important for the accurated description of the velocity field and the use of anisotropic turbulence models is prerequisite to more accurate flow field prediction.

\section{Conclusion}

Validation of the 3D finite element solver for the RANS equations with the Efficient $k-\epsilon$ turbulence model is conducted successfully using a high quality and high spatial resolution data set. The convection terms have been discretized using the Lagrange-Galerkin approach with advantage that, the CFL restriction is well performed. Moreover, addition of this characteristic method to the conservative form of the PDE and the implicit fractional step time stepping, allow to preserve the mass balance, the positivity of $k$ and $\epsilon$, as well as the stability of the scheme. In computed solutions, the weaker secondary currents were not reproduced, but it shlould be noticed that more refine turbulence modeling can produce improvement for such problem. Computations with and without non-hydrostatic are compared for the same trench to test the validity of the conventional hydrostatic pressure assumption. The model predicts reasonably the complex major features and the 3D flow tests were performed successfully against well-known unsteady non-uniform curved open channel flows. The non-hydrostatic pressure influence is found to be more significant with the increasing curvature region (e.g. cross-section $\alpha=120^{\circ}$ ). This suggest that the non-hydrostatic pressure may be useful and well suited for complicated geometry flows where its influence is thought to be significant. Further study is needed to improve the general applicability of the model, and the next stage of this work will be focus on the anisotropic turbulence-driven secondary motion.

\section{Acknowledgment}

The first author gratefully acknowledged Funding from the Swiss National Science Foundation through grant number 21-65095.01. Michel Deville, Alfio Quarteroni, Edie Miglio and Koen Blanckaert are acknowledged for their fruitfully discussions. 


\section{References}

1. Koen Blanckaert. Flow and turbulence in sharp open-channel bends. PhD thesis, Ecole polytechnique de Lausanne- EPFL, N0. 2545, 2002.

2. Xiabo Chao, Yafei jia, and Sam S. Y. Wang. Three-dimensional simulation of buoyant heat transfer in a curved open channel. In Proc. Int. Conf. on Advances in hydro-Science and-Engineering, ICHE, NCCHE Mississipi,USA, vol 6, pages 18-19\& on CDROM, 2004.

3. Thomas B. Gatski, M. Yousuff Hussaini, and John L. Lumley. Simulation and Modeling of Turbulent Flows. Oxford University Press, 198 Madison Avenue, New York, New York 10016, 1996.

4. Yong G. Lai, Larry J. Weber, and Virendra C. Patel. Three-dimensional model for hydraulic flow simulation. i: formulation and verification. ASCE, J. Hydr. Engrg., 129(3):196-205, 2003.

5. Michael A. Leschziner and Wolfang Rodi. Calculation of strongly curved open channel flow. ASCE, J. Hydr. Div., 103(10):1297-1314, 1979.

6. Célestin Leupi, Edie Miglio, Mustafa Altinakar, Alfio Quarteroni, and Michel Deville. Quasi-3d finite element shallow-water flow with $k-\varepsilon$ turbulence model. In Proc. Int. Conf. on Advances in hydro-Science and-Engineering, ICHE, NCCHE Mississipi, USA, vol 6, pages 400-401 \& on CDROM, 2004.

7. Patrick J. Luyten, John Eric Jones, Roger Proctor, Andy Tabor, Paul Tett, and Karen Wild-Allen. Coherens- a coupled hydrodynamical-ecological model for regional and shelf seas. Technical Report MUMM report, Management unit of the Mathematical Models of North Sea, 914pp, COSINUS, 1999.

8. Edie Miglio, Alfio Quarteroni, and Fausto Saleri. Finite element approximation of quasi-3D shallow water equations. Comput. Methods Appl. Mech. Engrg., 174(34):355-369, 1999.

9. Bijan Mohammadi and Olivier Pironneau. Analysis of $k$-epsilon Turbulence Model. Research in Applied Mathematics. John Wiley \& Sons, Chichester, 1994.

10. Nils Reidar B. Olsen. Three-dimensional cfd modeling of self-forming meandering channel. ASCE, J. Hydr. Engrg., 129(5):366-372, 2003.

11. Pierre Arnaud Raviart and Jean Marie Thomas. A mixed finite element method for 2nd order elliptic problems. Springer-Verlag, Mathematical Aspects of Finite Element Methods, Lecture notes in Methematics.

12. Wolfang Rodi. Turbulence models and their applications in hydraulics. Technical Report 2nd edition, IAHR, Delft, Netherlands, 1984.

13. Weiming $\mathrm{Wu}$, Wolfgang Rodi, and Thomas Wenka. 3D numerical modeling of flow and sediment transport in open channels. ASCE,J. Hydr. Engrg., 126(1):4-15, 2000 . 